\title{
Filigrane
}

Écoutes psychothérapiques

\section{Soigner les enfants violents, de Maurice Berger \\ Maurice Berger, 2012, Soigner les enfants violents, Paris, Dunod}

\section{Vincent Cardinal}

Volume 22, numéro 2, automne 2013

Psychanalyse et temporalités II

URI : https://id.erudit.org/iderudit/1022559ar

DOI : https://doi.org/10.7202/1022559ar

Aller au sommaire du numéro

Éditeur(s)

Revue Santé mentale au Québec

ISSN

1192-1412 (imprimé)

1911-4656 (numérique)

Découvrir la revue

Citer ce compte rendu

Cardinal, V. (2013). Compte rendu de [Soigner les enfants violents, de Maurice Berger / Maurice Berger, 2012, Soigner les enfants violents, Paris, Dunod].

Filigrane, 22(2), 105-110. https://doi.org/10.7202/1022559ar d'utilisation que vous pouvez consulter en ligne. 


\section{Soigner les enfants violents, de Maurice Berger ${ }^{1}$}

\section{Vincent Cardinal}

our son tout dernier livre paru en 2012, Soigner les enfants violents, Maurice Berger a choisi de répondre à l'appel de tous ceux qui, comme lui et son équipe, ont été un jour confrontés à la violence extrême de certains enfants et adolescents. Qu'ils soient cadres des hôpitaux, directeurs d'institutions scolaires et/ou de réadaptation, psychologues, infirmières et éducateurs, nombreux sont ceux et celles qui ont fait appel à lui pour se sortir de l'impasse dans laquelle les tentatives de soins de ces enfants les ont conduits. Or comment traiter cette violence chez des enfants qui mettent radicalement en échec toutes les méthodes de soins conventionnels? Voilà un des éléments fondamentaux de son dernier ouvrage qui s'inscrit par ailleurs dans la lignée de son ouvrage précédent - Voulons nous des enfants barbares? -, avec qui il partage une même communauté de pensée.

En fait, comme le dit Berger, devant la montée de la violence pathologique extrême chez enfants et les adolescents en France, « il n'est pas question de se payer de mots». Et nous pourrions ajouter à sa suite: «et de moyens pour y faire face et tenter de la traiter ». Car c'est précisément ce à quoi l'auteur, chef du département de pédopsychiatrie au CHU de St-Étienne en France depuis plus de trente-quatre ans, va s'attarder dans son ouvrage de près de trois cent pages.

Soigner les enfants violents se divise en trois parties. Dans la première, Maurice Berger nous présente une série de données cliniques récentes qui sont à la fois le fruit de son travail psychothérapeutique auprès des enfants et des adolescents violents, et à la fois le fruit d'un travail d'équipe sur le soin de ce type d'enfant en institution. D'entrée de jeu, l'auteur nous introduit au cas de Jason, un préadolescent particulièrement violent, qui éprouve fréquemment des «flashs» hallucinatoires au cours desquels il montre des potentialités meurtrières qui ont pu l'amener, par exemple, à torturer et à éviscérer des oisillons vivants. Sans entrer dans tous les détails de cette prise en charge individuelle qui met à rude épreuve son vécu contre transférentiel, Berger nous montre, au 
fil de son récit, toute la complexité des processus psychiques impliqués dans la lente transformation de la personnalité de ce jeune garçon (deux ans de travail ininterrompu à six séances de psychothérapie psychanalytique par semaine). Un des principaux constats auxquels lui et son équipe arrivent, c'est qu'un travail psychothérapique individuel avec ce type d'enfant, s'il n'est pas accompagné d'un dispositif de soin groupal et institutionnel suffisamment solide, est foncièrement voué à l'échec. Berger est convaincu que si ces enfants (ou ces adolescents) ne sont pas traités, ou s'ils le sont insuffisamment, ils n'auront d'autres « choix » que de répéter cette violence pathologique extrême et ce faisant, de faire de nouvelles victimes. Berger et son équipe y vont aussi d'un constat surprenant: c'est d'abord en empêchant l'enfant d'utiliser sa violence, en la «contenant» selon diverses modalités que l'auteur va préciser tout au long de son livre, qu'une équipe de soin est la mieux placée pour faire naître chez l'enfant ce qu'il appelle une pensée différenciée. Cette dernière consiste en la capacité de différencier ce qui vient de lui de ce qui vient d'autrui, de distinguer les images du passé par rapport au présent, et d'identifier ce qu'il ressent, de le mettre en mot et de le partager. Or dans les milieux «psy» c'est plutôt l'opinion inverse qui a cours généralement. En effet, pour un nombre considérable de professionnels en santé mentale, du moins au Québec, la croyance veut que ce soit d'abord en aidant l'enfant à penser (sur lui-même) qu'on peut l'aider à contenir sa violence. En réalité, les travaux de Berger et de son équipe nous démontrent plutôt le contraire. L'auteur précise cependant que la prise en charge de cette violence ne peut se faire sans une double articulation de l'intervention elle-même. L'un ne va pas sans l'autre: écouter un enfant violent sans contenance préalable est inefficace, mais la contenance seule, sans mettre en place un dispositif d'écoute spécifique, est tout aussi inutile. Il faut donc à la fois contenir sa violence pour l'aider à penser, et faire advenir sa pensée pour que les actes violents ne se reproduisent plus.

Toujours dans cette première partie, Berger établit un lien très clair entre la négligence vécue en bas âge, c'est-à-dire avant l'apparition du langage, et le développement des formes de violence pathologique extrême chez l'enfant. Il développe le concept de traumatismes relationnels répétitifs pour décrire l'effet de la négligence sur son développement affectif, social et cognitif. Il va ainsi reprendre le concept de négligence et montrer comment cette négligence affecte l'enfant dans ses besoins de base, besoins qu'il va définir de la façon suivante: «D'une manière générale, la négligence est une absence d'attention portée aux besoins élémentaires du nourrisson, besoin de stabilité, de prévisibilité, de stimulation, absence qui s'accompagne souvent d'une déformation 
des messages que l'enfant émet» (65). C'est donc à l'occasion de ces traumatismes relationnels précoces ${ }^{2}$ que vont s'instaurer selon Berger les différents éléments qui vont paver la voie aux différentes formes de violence qu'il a repérées dans sa clinique de l'enfant et l'adolescent. Dans sa perspective, la violence pathologique extrême (VPE) est la résurgence de ces traumas relationnels précoces qui ont été intériorisés à la période préverbale sous la forme de sensations corporelles et/ou d'images à l'état brut. En fait, Berger va introduire dans Soigner les enfants violents une différence qu'il ne faisait pas encore dans son livre précédent. Si la VPE est une forme de violence indifférenciée quant à son but et à son objet, dans la VAS (violence avec processus sadique), c'est le contraire qui se passe: le jeune sujet organise un scénario d'attaque délibéré envers l'autre dont il vise intentionnellement l'un des points de vulnérabilité. Si la VPE surgit sous la forme d'un flash hallucinatoire dont le sujet ne se souvient plus lorsque «la crise» est terminée, à l'opposé, dans la VAS, l'enfant éprouve du plaisir à la vue de la souffrance de l'autre. Le statut de «l'objet » y est donc différent, et la VAS met les professionnels en très grande difficulté car elle constitue une triple pénétration agie, à la fois sur le dispositif de soin, sur leur état affectif et, ultimement, sur leur travail de théorisation.

Enfin, Berger identifie trois autres formes de violence qu'il décrit comme la violence passionnelle, la violence intrafamiliale pour laquelle un placement de l'enfant peut parfois devenir nécessaire, et la violence au sein des relations fraternelles. Comme à chaque fois, Berger nous propose une vignette clinique qui vient éclairer son propos. Et comme à chaque fois, on ne peut manquer d'être fasciné par la pensée de ce clinicien. Les quelques pages qu'il nous offre sur son travail auprès de Lucien et de ses frères, l'histoire d'un inceste entre frères, nous permet de la voir se déployer dans toute sa finesse, à travers sa compréhension des mouvements pulsionnels de l'adolescent et de sa fratrie.

La deuxième partie de l'ouvrage pourrait être considérée comme le cœur de son élaboration. C'est dans cette partie que Berger va préciser avec force détails tout le dispositif qu'ils ont mis en place son équipe et lui pour s'attaquer à cette violence extrême de l'enfant. Berger y décrit un univers de soin constitué de différentes « enveloppes » qui sont à la fois institutionnelle, groupale et individuelle. Ces enveloppes ont une fonction de contenance qui va faire en sorte que le travail psychique soit éventuellement possible. À cet égard, Berger prend bien soin de préciser que «la contenance passe par l'établissement d'un cadre», lequel « doit être stable et solide» (149). Par ailleurs, l'idée fondamentale qui sous-tend son dispositif est de réunir les conditions favorables pour produire de la matière à penser, du "psychisable», chez des 
enfants pour qui l'absence de tout sentiment de culpabilité et l'expulsion hors de soi de tout vécu traumatique constituent une barrière infranchissable à l'établissement d'un lien thérapeutique.

Toujours dans le même sens, on pourrait se représenter l'ensemble des «enveloppes» de son dispositif comme une série de poupées gigognes qui s'emboîtent les unes dans les autres. Ces «enveloppes» peuvent avoir à la fois un aspect matériel et immatériel, et l'une de ces premières enveloppes est justement le cadre institutionnel. Berger réfere ici à l'aspect matériel de l'institution avec ses murs et ses différents espaces de vie et de travail; mais il réfère aussi à son aspect médical et administratif qu'il va mettre à profit dans son travail auprès des enfants et des adolescents violents. Dans son livre, il donne une place centrale à ce qu'il appelle «le projet de service», rédigé avec précision, duquel émane un cadre de soins adapté pour chaque enfant. Berger compare le "projet de service » à une forme de boussole qui fixe les grands objectifs à atteindre, ce qui dans le cas de son service équivaut aux objectifs thérapeutiques de l'équipe soignante. L'auteur nous indique que ces objectifs servent en fait de «base de travail» pour toute son équipe, et que c'est ce qui permet à cette dernière de garder une forme de cohérence et de stabilité à travers le temps.

Le second aspect de ces "conteneurs institutionnels", comme il les appelle, est l'établissement d'un cadre de soins précis pour chaque enfant. L'équipe du $\mathrm{D}^{\mathrm{r}}$ Berger apporte un soin méticuleux à l'évaluation des besoins de chacun des enfants du service. Puis, elle met en place un protocole de soins qui codifie un certain nombre de choses dans la vie des enfants et des adolescents hospitalisés. Par exemple, le temps de contention ou de retrait en chambre est inscrit au plan de soin, discuté en groupe et présenté à l'enfant par le chef de service. De même, les enveloppements ou les bains thérapeutiques, qui sont une manière de faire vivre à l'enfant une expérience sensorielle qui sous-tend la constitution de son enveloppe corporelle, sont étroitement codifiés et planifiés par les membres de l'équipe. Enfin, Berger expose ses vues sur la prise de médicament, l'isolement et la parole, comme autant de moyens mis en place pour favoriser le développement d'une pensée différenciée chez l'enfant et l'aider à faire face à sa violence interne.

Par ailleurs, nous ne voudrions pas passer sous silence une réflexion intéressante que fait l'auteur sur le travail à l'urgence lors d'une situation de crise familiale et le travail en interdisciplinarité dans le champ de la protection de l'enfance. Malheureusement, faute d'espace, il nous semble judicieux d'inviter le lecteur à se référer au livre afin d'avoir accès à tout le détail et à la complexité de ces propositions. 
Dans la troisième et dernière partie de son ouvrage, Berger reprend son bâton de pèlerin et fait voler en éclats la thèse voulant que la dernière version de la Loi française sur la protection de la jeunesse constitue une avancée majeure pour la protection des enfants et des adolescents. À moins d'être vivement intéressé par toutes ces questions, l'intérêt du lecteur québécois y sera probablement moindre. Mais il faut reconnaître à Maurice Berger le mérite d'avoir attiré notre attention sur une dimension souvent négligée lorsqu'il est question de la protection de l'enfance: celle du développement psychique de l'enfant. En effet, Berger affirme avec raison que nous ne sommes sans doute pas prêts à penser la protection de l'enfance en dehors des macrotraumatismes que sont les abus physiques, les abus sexuels et les formes de négligence extrême. La critique très dure qu'il formule à l'endroit de la Loi française de la protection de la jeunesse se fonde sur l'idée qu'il existe d'autres formes de traumatismes, moins « visibles». Ces autres modalités du traumatisme, qu'il a repérées en tant que traumatismes relationnels précoces, sont ainsi très peu considérées en France dans la loi de 2007. À l'instar de ce qui se fait dans la Loi de la protection de la jeunesse au Québec, Berger voudrait que la législation française tienne compte véritablement de la maltraitance psychologique plutôt que de s'enorgueillir de principes qui font la part trop belle à la symétrie des droits entre les parents et les enfants. Dans cette optique, si l'auteur fait une place enviable à la Loi de la Protection de la jeunesse québécoise dans son livre, notre longue expérience en ce domaine et notre position d'observateur privilégié de la scène québécoise de la protection de la jeunesse nous a parfois fait douter de certaines affirmations qui y sont faites. Par exemple, lorsqu'il cite les principes qui sont au cœur des visites médiatisées au Québec ${ }^{3}$, nous ne sommes pas du tout certain que ces principes soient effectivement bien compris, ni même appliqués. D’ailleurs, le concept de «visites médiatisées » est certainement, à notre avis, l'un des plus galvaudés au Québec. Ceci dit, Maurice Berger reste pour nous un auteur remarquable doté d'un sens clinique hors du commun. Son dernier livre en est certainement une preuve supplémentaire.

Vincent Cardinal

Centre de santé et de services sociaux Ahuntsic Montréal-Nord

1165, boul. Henri-Bourassa Est Montréal, Q.C., H2C 3K2 vincent.cardinal.csssamn@ssss.gouv.qc.ca 


\section{Notes}

1. Maurice Berger, 2012, Soigner les enfants violents, Paris, Dunod.

2. Berger utilise parfois les deux expressions pour désigner la même chose.

3. «Les contacts entre parents et enfants doivent en tout temps répondre aux besoins et aux intérêts de l'enfant et non à ceux des parents. Les modalités de contact se fixent en se basant sur les réactions et le développement de l'enfant. » p. 254 